\title{
Traducción y adaptación de la Level of Personal Functioning Scale - Brief Form 2.0 \\ Translation and adaptation of the Level of Personal Functioning Scale - Brief Form 2.0
}

\section{Tradução e adaptação da Level of Personal Functioning Scale - Brief Form 2.0}

\author{
Christian Schetsche, ORCID 0000-0002-6353-3571 \\ Universidad de Buenos Aires, Argentina
}

\begin{abstract}
Resumen: La quinta edición del Manual Diagnóstico y Estadístico de los Trastornos Mentales (DSM-5) introdujo el concepto de niveles de funcionamiento de la personalidad, los cuales indican la gravedad del trastorno de la personalidad. En nuestro conocimiento, no existen instrumentos psicométricos concisos para la evaluación del nivel de funcionamiento de la personalidad en lengua castellana, por lo que el objetivo del presente estudio consistió en la traducción y adaptación de la Level of Personal Functioning Scale - Brief Form 2.0. La muestra $(\mathrm{N}=361)$ se recogió por internet. Los resultados del análisis factorial confirmatorio mostraron que el instrumento tiene una estructura bifactorial y que puede utilizarse, además de sus dos dimensiones autofuncionamiento y funcionamiento interpersonal, una dimensión que representa el funcionamiento general. Las consistencias internas obtuvieron valores aceptables, y en el análisis de validez convergente se encontraron correlaciones significativas con todos los rasgos de la personalidad.
\end{abstract}

Palabras clave: nivel de funcionamiento de la personalidad; análisis factorial confirmatorio; traducción; adaptación.

Abstract: The fifth edition of the Diagnostic and Statistical Manual of Mental Disorders (DSM-5) introduced the concept of levels of personality functioning, which indicates the severity of personality disorders. In our knowledge, there are no concise psychometric instruments for assessing the level of personality functioning in Spanish, so the objective of this study was the translation and adaptation of the Level of Personal Functioning Scale Brief Form 2.0. The sample $(\mathrm{N}=361)$ was collected via internet. The results of the confirmatory factor analysis showed that the instrument has a bifactorial structure and it can be used, in addition to its two dimensions, self-functioning and interpersonal functioning, a factor that represents general functioning. Evidence of internal consistency is provided, and significant correlations were found with all personality traits.

Keywords: level of personal functioning; confirmatory factor analysis; translation; adaptation. 
Resumo: A quinta edição do Manual Diagnóstico e Estatístico de Transtornos Mentais (DSM-5) introduziu o conceito de níveis de funcionamento da personalidade, que indicam a gravidade do transtorno da personalidade. Tanto quanto sabemos, não existem instrumentos psicométricos concisos para avaliar o nível de funcionamento da personalidade na língua espanhola, pelo que o objetivo deste estudo consistiu na tradução e adaptação da Level of Personal Functioning Scale - Brief Form 2.0. A amostra $(\mathrm{N}=361)$ foi coletada via internet. Os resultados da análise fatorial confirmatória mostraram que o instrumento possui uma estrutura bifatorial e que pode ser utilizado, para além das suas duas dimensões, autofuncionamento e funcionamento interpessoal, com um fator que representa o funcionamento geral. As consistências internas obtiveram valores aceitáveis, e na análise da validade convergente foram encontradas correlações significativas com todos os traços de personalidade.

Palavras-chave: nível de funcionamento da personalidade; análise fatorial confirmatória; tradução; adaptação.

Recibido: 11/12/2020

Aceptado: 30/04/2021

Cómo citar:

Schetsche, C. (2021). Traducción y adaptación de la Level of Personal Functioning Scale Brief Form 2.0. Ciencias Psicológicas, 15(2), e-2387. doi: https://doi.org/10.22235/cp.v15i2.2387

Correspondencia: Christian Schetsche, Universidad de Buenos Aires, Argentina. E-mail: christianschetsche@psi.uba.ar

Para la realización de un psicodiagnóstico, se tiene en cuenta una determinada secuencia de pasos a seguir. En el nivel uno, se selecciona un sistema de clasificación determinado (diagnóstico categórico) que podría estar basado en el ICD o DSM. A continuación, se analizan las facetas clínicamente relevantes, quiere decir que se realiza una evaluación general, la verificación de síntomas (por ejemplo, la depresión y la ansiedad) y los aspectos de la personalidad. En el nivel tres, se utilizan procedimientos que, más allá de la patología y los aspectos asociados, cubren áreas clínicamente relevantes en el sentido de consecuencias del trastorno, como las alteraciones en la vida cotidiana y/o la calidad de vida. Por último, en el nivel cuatro, se requiere una diferenciación con respecto a las escuelas de terapia, ya que los procedimientos de diagnóstico difieren según la terapia que se esté llevando a cabo (Laireiter, 2013; Stieglitz, 2014).

De esta manera, analizar a las personas y determinar aquellas diferencias que son relevantes para la planificación y el éxito de la psicoterapia consisten en unos de los principales desafíos de la investigación clínica de la psicología (Levy \& Clarkin, 2003). Existe un consenso cada vez mayor en que la gravedad de un posible trastorno de la personalidad es una variable del paciente que es muy relevante para el diagnóstico (Bernstein, 1998; Hopwood et al., 2011; Leising \& Zimmermann, 2011). Esto se ejemplifica en el área 
de diagnóstico del trastorno de personalidad: aunque los sistemas anteriores DSM-IV (American Psychiatric Association [APA], 2000) e ICD-10 (World Health Organization [WHO], 1993) mencionaban distintas categorías de trastornos de personalidad, no clasificaban sus gravedades.

En mayo de 2013, se publicó la quinta edición del Manual Diagnóstico y Estadístico de los Trastornos Mentales (DSM-5). Mientras que la sección II (Elementos Esenciales: Criterios y Códigos de Diagnóstico) fue adoptada prácticamente sin cambios del modelo anterior (el DSM-IV), el DSM-5 contiene, en la sección III (Medidas y modelos emergentes), un modelo alternativo que complementa la clasificación de los trastornos de la personalidad (APA, 2013).

La innovación central de este modelo alternativo consiste en la diferenciación de dos componentes del trastorno de la personalidad: por un lado, están las deficiencias en el nivel funcional de la personalidad (criterio A) y, por otro, la presencia de rasgos de personalidad desadaptativos (criterio B; Zimmermann et al., 2013).

A través del criterio A, se evalúan las deficiencias genéricas que subyacen a todos los tipos de trastornos de personalidad. Este enfoque supone que todos los trastornos de personalidad comparten algunos puntos esenciales que, en su conjunto, los distinguen de otras condiciones mentales (Morey et al., 2011). El nuevo modelo del DSM-5 (APA, 2013) operacionaliza el nivel funcional de la personalidad a través de la Level of Personality Functioning Scale (LPFS), de Bender, Morey y Skodol (2011). Esta escala tiene la tarea de aclarar si existe un trastorno de personalidad y, en caso afirmativo, evaluar su magnitud. La escala se basa en la suposición de que el denominador común de todos los trastornos de personalidad radica en el deterioro de las capacidades básicas de adaptación (Livesley, 1998) y tiene las siguientes dimensiones y subdimensiones (APA, 2013): autofuncionamiento (identidad y autodirección) y funcionamiento interpersonal (empatía e intimidad). La suposición de que el trastorno de personalidad es esencialmente un trastorno del "yo" y las relaciones interpersonales están en línea con una amplia gama de teorías diferentes e influyentes sobre el desarrollo y el tratamiento de los trastornos de la personalidad (Hopwood, Schade, Krueger, Wright \& Markon, 2013; Kernberg, 2012; Luyten \& Blatt, 2011).

En consecuencia, el criterio A facilita la determinación si el paciente tiene un trastorno de la personalidad y la evaluación de su gravedad, mientras que el criterio B (los rasgos de la personalidad) suministra la información sobre las características únicas del desorden (Bach \& Hutsebaut, 2018). Debido a eso, el modelo puede entenderse como una construcción híbrida basada en la evaluación de las dos dimensiones mencionadas. El enfoque doble de la APA (2013) tiene el propósito de asegurar la continuidad con la práctica diagnóstica previa y, al mismo tiempo, crear la base para un nuevo paradigma de diagnóstico clínico de la personalidad (Zimmermann et al., 2013).

A pesar de su relativa novedad, ya han surgido críticas al modelo hibrido que propone el DSM-5. En una muestra de reclusas, Sleep, Wygant y Miller (2018) mostraron que el nivel funcional de la personalidad contribuyó a la predicción del trastorno límite de la personalidad, del trastorno de personalidad del tipo narcisista y de las características afectivas interpersonales de la psicopatía, pero que el nivel funcional no contribuyó a la predicción del trastorno de personalidad del tipo antisocial y las características impulsivas-antisociales de 
la psicopatía. En estos casos, los rasgos de la personalidad continuaban teniendo mayor capacidad explicativa.

Por otro lado, cabe destacar que estos resultados entran en conflicto con los estudios que sugieren que la gravedad general del trastorno de personalidad proporciona información adicional por encima de los rasgos de la personalidad (Calabrese \& Simms, 2014; Hopwood, Thomas, Markon, Wright \& Krueger, 2012).

Con el fin de evaluar el nivel de funcionamiento de la personalidad, se han desarrollado numerosos instrumentos: el SIPP-118 de Verheul et al. (2008), el General Assessment of Personality Disorder (GAPD) de Hentschel y Livesley (2013) y, en lengua castellana, el OPS-SQ con un total de 95 ítems los cuales corresponden a 8 subescalas (De la Parra et al., 2018) y la Level of Personality Function Scale de Stover y Bruno (2019) con un total de 80 ítems.

Con respecto a instrumentos breves con menos de 20 ítems, existen en lengua alemana el Inventory of Personality Organization (IPO) de Lenzenweger, Clarkin, Kernberg y Foelsch (2001), en lengua alemana e inglesa el OPD-SFK (Ehrenthal et al., 2012, 2015) y, en inglés, la Level of Personal Functioning Scale - Brief Form 2.0 (LPFS - BF 2.0) de Weekers, Hutsebaut y Kamphuis (2019).

En nuestro conocimiento, no existe un cuestionario breve en castellano que esté específicamente orientado al nivel de funcionamiento de la personalidad del DSM-5. La existencia de un instrumento de dichas características facilitaría no solo la posibilidad de realizar una primera evaluación rápida, sino también la profundización en la investigación de los trastornos de la personalidad a través de estudios extensos que requieren cuestionarios concisos. Es por ello que el objetivo del presente estudio consistió en la validación de la Level of Personal Functioning Scale - BF 2.0 de Weekers et al. (2019) y, debido a la correlación elevada entre las dimensiones autofuncionamiento y funcionamiento interpersonal (Bach \& Hutsebaut, 2018) en la evaluación de su estructura factorial.

\section{Método}

\section{Cumplimiento con estándares éticos}

Esta investigación fue aprobada por el Comité de Conductas Responsables de la Universidad de Buenos Aires.

\section{Procedimiento y muestra}

Para la recolección de datos, se utilizó la plataforma digital de Google Forms@. En la página inicial del cuestionario, se informó sobre la participación anónima y el trato confidencial de la información de acuerdo con la Ley n. ${ }^{\circ}$ 25.326. Asimismo, se comunicó la posibilidad de retirarse en cualquier momento de la investigación y, después de aceptar participar mediante el consentimiento informado, se presentaron los cuestionarios. En caso de que los sujetos tuvieran inconvenientes o dudas durante las respuestas, se dejó el email de contacto del investigador. El reclutamiento de los participantes se realizó a través de las redes sociales de Facebook, Instagram y WhatsApp, y, con el fin de asegurar una realización satisfactoria de la encuesta, se llevó a cabo una prueba piloto con 30 individuos. 
Se realizó un muestreo no-probabilístico y por bola de nieve. La muestra se recolectó entre el 02 de octubre y el 05 de noviembre de 2020 y estuvo conformada por 361 adultos $(\mathrm{M}$ edad= 41.01, $\mathrm{DE}=15.58$; femenino= 203) residentes en Argentina (30\% de la Ciudad Autónoma de Buenos Aires, $22 \%$ de Gran Buenos Aires, $11 \%$ de la Provincia de Buenos Aires y $38 \%$ de otras provincias de Argentina). El $72 \%$ eran de nivel universitario incompleto o superior.

\section{Instrumentos}

Level of Personal Functioning Scale - Brief Form 2.0.

La Level of Personal Functioning Scale - Brief Form 2.0 (LPFS - BF 2.0) fue desarrollada por Weekers et al. (2019) y evalúa las dos dimensiones: autofuncionamiento y funcionamiento interpersonal, y, a través del total de sus 12 ítems, puede evaluarse el funcionamiento general. Para contestar, se utiliza una escala Likert de cuatro puntos que va desde 1 = Totalmente en desacuerdo hasta $4=$ Totalmente de acuerdo. A mayor puntaje mayor disfuncionamiento personal. En el estudio original, obtuvo unas consistencias internas $.82 \geq \alpha \geq .71$.

La traducción al castellano fue realizada por tres psicólogos bilingües y posteriormente se realizó la traducción inversa. Este procedimiento permitió debatir la idoneidad de las traducciones realizadas y así seleccionar aquellas que mejor se adaptaran a los ítems correspondientes de la versión original.

Además, debe resaltarse que no se ha utilizado una escala Likert de cuatro puntos, tal como lo proponen los autores del instrumento, sino una escala Likert de cinco puntos que abarca los valores $0=$ Totalmente en desacuerdo hasta $5=$ Totalmente de acuerdo. Esa modificación se debe a que, durante la prueba piloto del estudio, un elevado número de participantes manifestó su deseo de marcar una respuesta de valor intermedio.

\section{Mini-IPIP}

Se utilizó la validación argentina que Simkin, Borchardt Dutera y Azzollini (2020) realizaron del Compendio Internacional de Ítems de Personalidad Abreviado (Mini International Personality Item Pool, Mini-IPIP). El instrumento tiene 20 ítems que corresponden a 5 rasgos de la personalidad. Para su contestación, se utiliza una escala Likert de cinco puntos $(1=$ Completamente en desacuerdo hasta $5=$ Completamente de acuerdo $)$. Los autores del mencionado estudio obtuvieron consistencias internas entre $0,77 \leq \omega \leq .88 \mathrm{y}$, en el presente estudio, estas alcanzaron los siguientes valores: apertura a la experiencia $(\omega=$ $.74)$, responsabilidad $(\omega=.77)$, extraversión $(\omega=.75)$, amabilidad $(\omega=.83)$ y neuroticismo $(\omega=.75)$.

\section{Análisis de datos}

A través del método Minimum Covariance Determinant que fue desarrollado por Leys, Klein, Dominicy y Ley (2018), se realizó la detección de valores atípicos multivariante. Para ello, se sirvió del paquete MASS de Venables y Ripley (2002). La comprobación de la normalidad multivariante se realizó a través del test de Mardia (1970) y el MVN de Korkmaz, Goksuluk y Zararsiz (2014). En consonancia con el Psych de Revelle (2019), se realizaron el análisis factorial exploratorio, el cálculo de los índices de complejidad de Hofmann (1978), 
de las consistencias internas ( $\alpha$ y $\omega$ ) y de las correlaciones parciales de Spearman. A través del Lavaan de Rosseel (2012), se llevaron a cabo el análisis factorial confirmatorio y la invarianza factorial. Todos los paquetes mencionados forman parte del software $\mathrm{R}$ de Core Team (2020) y, para todos los cálculos, se utilizó el valor de probabilidad $\mathrm{p} \leq .05$.

\section{Resultados}

\section{Valores atípicos y normalidad multivariante}

Un total de 23 observaciones fueron clasificadas como valores atípicos, por lo que se excluyeron de la muestra, resultando esta en 338 sujetos (188 del género femenino) y, a través del test de Mardia (1970), se observó que los ítems no representaban una normalidad multivariante.

\section{Análisis factorial exploratorio}

En caso de incumplir con el supuesto de normalidad multivariante, Fabrigar, Wegener, MacCallum y Strahan (1999) sugieren utilizar para el análisis factorial exploratorio el método de los ejes principales. Además, se utilizó un análisis paralelo y método de rotación Oblimin (Costello \& Osborne, 2005). La evaluación del Scree-Plot justificó la extracción de 2 factores, lo cual está en consonancia con la estructura factorial que proponen Weekers et al. (2019). La Tabla 1 muestra las cargas factoriales y los índices de complejidad de Hofmann (1978) del modelo resultante del análisis factorial exploratorio, el cual explica el $47 \%$ de la varianza. Como puede observar, los ítems 10 y 11 podrían considerarse como críticos debido a que sus cargas factoriales son $\leq .50$ (Hair, Black, Babin \& Anderson, 2010) y porque tienen índices de complejidad de Hofmann (1978) que son considerablemente más altos que los demás. Además, el ítem 11 carga más en autofuncionamiento que en funcionamiento personal. Al comparar estos resultados con los que obtuvieron Bach y Hutsebaut (2018) se observó que los mencionados autores encontraron las mismas características con relación a estos ítems.

Aunque la realización de un análisis factorial exploratorio y un análisis factorial confirmatorio en la misma muestra pueda conducir a un sobreajuste en la evaluación de la estructura interna (Fokkema \& Greiff, 2017), se decidió continuar con el análisis factorial confirmatorio. Esa decisión se debió a la similitud con la estructura factorial encontrada por Bach y Hutsebaut (2018), quiere decir que el análisis factorial exploratorio del presente estudio no encontró una estructura factorial nueva, sino que reconfirmó la estructura ya encontrada en un estudio previo. 
Tabla 1.

Cargas factoriales e índices de complejidad de Hofmann (1978) del modelo según el resultado del análisis factorial exploratorio

\begin{tabular}{|c|c|c|c|c|}
\hline $\begin{array}{l}\text { Número } \\
\text { ítem }\end{array}$ & Frase & $\mathrm{AF}$ & FI & $\mathrm{ICH}$ \\
\hline LPFS_01 & A menudo, no sé quién soy realmente. & .797 & & 1.002 \\
\hline LPFS_02 & A menudo, pienso de manera muy negativa sobre mí mismo(a). & .934 & & 1.043 \\
\hline LPFS_03 & Mis emociones cambian sin que pueda controlarlas. & .617 & & 1.126 \\
\hline LPFS_04 & No tengo idea qué hacer con mi vida. & .695 & & 1.001 \\
\hline LPFS_05 & A menudo, no entiendo mis propios pensamientos y sentimientos. & .681 & & 1.093 \\
\hline LPFS_06 & A menudo, me impongo exigencias poco realistas. & .587 & & 1.037 \\
\hline LPFS_07 & $\begin{array}{l}\text { A menudo, tengo dificultades para entender los pensamientos y } \\
\text { sentimientos de otros. }\end{array}$ & & .701 & 1.018 \\
\hline LPFS_08 & $\begin{array}{l}\text { A menudo, encuentro difícil soportarlo cuando otros tienen una opinión } \\
\text { diferente a la mía. }\end{array}$ & & .632 & 1.017 \\
\hline LPFS_09 & $\begin{array}{l}\text { A menudo, no entiendo del todo por qué mi comportamiento tiene } \\
\text { cierto efecto en otros. }\end{array}$ & & .586 & 1.025 \\
\hline LPFS_10 & Mis relaciones y amistades nunca duran mucho. & & .286 & 1.292 \\
\hline LPFS_11 & $\begin{array}{l}\text { A menudo, me siento muy vulnerable cuando las relaciones se vuelven } \\
\text { más personales. }\end{array}$ & .453 & & 1.527 \\
\hline LPFS_12 & $\begin{array}{l}\text { A menudo, no logro cooperar bien con otros de una manera que sea } \\
\text { satisfactoria para ambos. }\end{array}$ & & .588 & 1.027 \\
\hline
\end{tabular}

Notas: $\mathrm{n}=338$; AF, autofuncionamiento; FI: funcionamiento interpersonal; ICH: índice de complejidad de Hofmann (1978).

\section{Análisis factorial confirmatorio}

Basado en lo expresado anteriormente, se evaluaron inicialmente dos modelos: el LPFS-BF 2.0 - Original representa la estructura factorial propuesta por Weekers et al. (2019) y el LPFS-BF 2.0 - EFA el modelo según el resultado del análisis factorial exploratorio.

Debido al incumplimiento con el supuesto de normalidad multivariante, se utilizaron los índices ajustados S-B $\chi 2$ de Satorra y Bentler (2000) para la evaluación de los modelos, ya que estos utilizan un error estándar robusto (Yu, 2002). Siguiendo las sugerencias de $\mathrm{Hu}$ y Bentler (1999), los siguientes valores representan un ajuste de modelo adecuado: $\chi 2 / \mathrm{df} \leq$ $3, \mathrm{RMSEA} \leq .06, \mathrm{SRMR} \leq .08, \mathrm{CFI} \geq .95, \mathrm{TLI} \geq .95$. Teniendo en cuenta estos valores, se puede observar en la Tabla 2 que el modelo resultante al análisis factorial exploratorio obtuvo los índices de ajuste más favorables. Aun así, se advirtió que, con excepción del SRMR, todos los índices se encontraron por debajo de los valores sugeridos por Hu y Bentler (1999). 
Tabla 2.

Índices de ajuste de los modelos competidores

\begin{tabular}{lccccccccc}
\hline Modelos competidores & $\chi^{2}$ MLM & df & $\chi^{2 / d f}$ & Scaling & p & RMSEA (90\% CI) & SRMR & CFI & TLI \\
\hline LPFS-BF 2.0 - & 180.291 & 53 & 3.402 & 1.227 & 0.000 & $0.093(0.079-0.109)$ & 0.063 & 0.903 & 0.879 \\
Original & 162.677 & 53 & 3.069 & 1.202 & 0.000 & $0.086(0.071-0.101)$ & 0.055 & 0.918 & 0.898 \\
LPFS-BF 2.0 - EFA & 162
\end{tabular}

Notas: $\mathrm{n}=338 ; \chi 2$ MLM: Chi-cuadrado utilizando el método de máxima verosimilitud con errores estándares robustos (MLM); df: grados de libertad; Scaling: factor de ajuste de la corrección de Satorra-Bentler; RMSEA: error cuadrático medio de aproximación; SRMR: residuo estandarizado cuadrático medio, CFI: índice de ajuste comparativo; TLI: Tucker Lewis Index.

\section{Reducción de ítems}

Con el fin de realizar una purificación de medida y alcanzar unos valores aceptables, se analizaron las correlaciones de los residuos estandarizados. De acuerdo a las sugerencias de Hair et al. (2010), aquellos ítems cuyos residuos estandarizados exhiben una gran cantidad de $>|4.00|$ con otros ítems pueden considerarse como críticos. Además, aconsejan una revisión atenta de aquellos ítems con una gran cantidad de correlaciones entre $|2.00|$ y $|4.00|$ con otros. A raíz de eso, los ítems 2, 6, 10 y 11 fueron eliminados, por lo que la versión final comprendió un total de 8 ítems, es decir 4 por cada factor.

Siguiendo el segundo objetivo del estudio, se contrastaron 3 estructuras factoriales: de 2 factores (autofuncionamiento y funcionamiento interpersonal) correlacionados (LPFSBF 2.0 - 8), con un solo factor de funcionamiento general (LPFS-BF 2.0 - 8 UNI) y con una estructura bifactorial (LPFS-BF 2.0 - 8 BI), la cual evalúa independientemente los 2 factores mencionados y, simultáneamente, el factor de funcionamiento general. La Figura 1 representa los tres modelos que fueron evaluados. 


\section{a) Modelo de dos factor correlacionados}

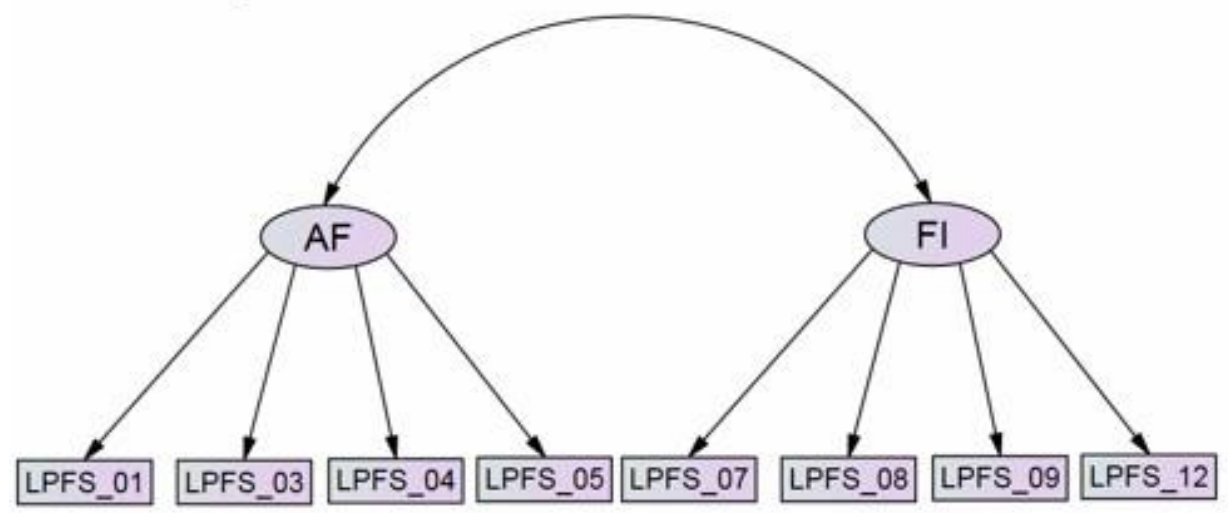

b) Modelo unifactorial

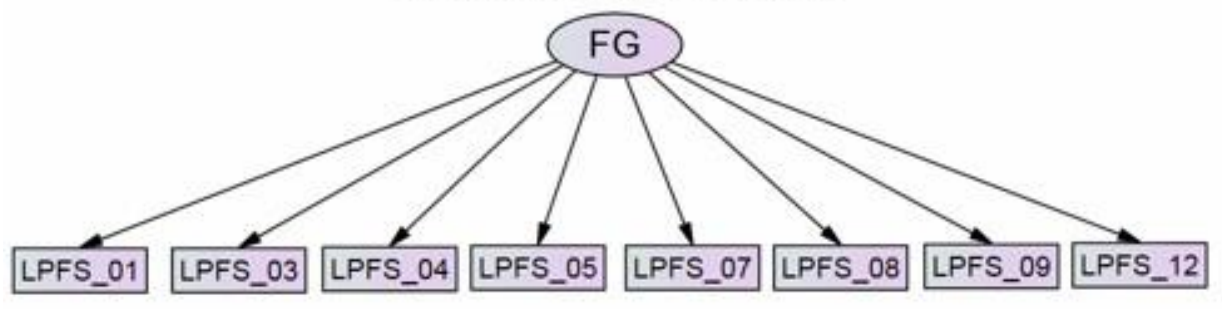

c) Modelo bifactorial

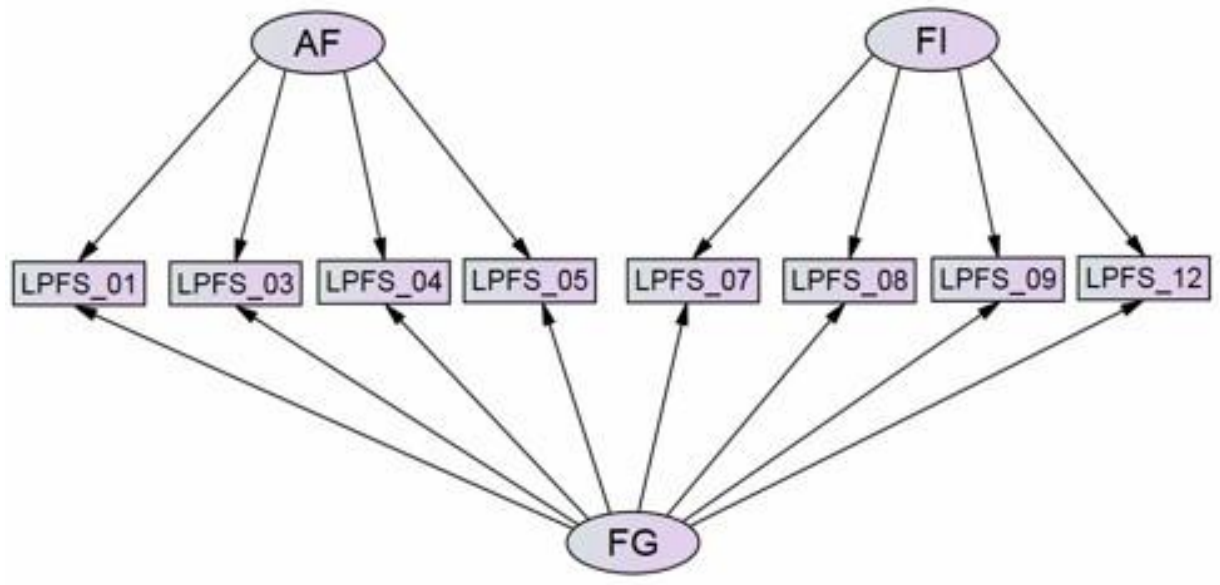

Figura 1. Representaciones gráficas de los modelos analizados

Notas: Modelos de medida y de estructura de los tres modelos competidores del LPFS-BF 2.0-8; AF: autofuncionamiento; FI: funcionamiento interpersonal; FG: funcionamiento general. 
La Tabla 3 representa los índices de ajuste de los tres modelos con 8 ítems. Por un lado, puede observarse que el modelo unifactorial obtuvo los valores más bajos y, por otro lado, que todos los índices del modelo de 2 factores correlacionados y del modelo bifactorial se encontraron dentro de unos rangos adecuados según las indicaciones de $\mathrm{Hu}$ y Bentler (1999). Así mismo, los resultados indican una ligera superioridad del modelo bifactorial.

Tabla 3.

Índices de ajuste de los modelos competidores

\begin{tabular}{lccccccccc}
\hline $\begin{array}{l}\text { Modelos } \\
\text { competidores }\end{array}$ & $\chi 2^{\text {MLM }}$ & $\mathrm{df}$ & $\chi 2 / \mathrm{df}$ & Scaling & $\mathrm{p}$ & RMSEA (90\% CI) & SRMR & CFI & TLI \\
\hline LPFS-BF 2.0 - 8 & 28.702 & 19 & 1.511 & 1.203 & 0.071 & $0.043(0.000-0.073)$ & 0.031 & 0.987 & 0.981 \\
LPFS-BF 2.0 - 8 & 128.173 & 20 & 6.409 & 1.269 & 0.000 & $0.143(0.120-0.167)$ & 0.090 & 0.851 & 0.792 \\
UNI & 15.916 & 12 & 1.326 & 1.187 & 0.195 & $0.034(0.000-0.074)$ & 0.020 & 0.995 & 0.988 \\
$\begin{array}{l}\text { LPFS-BF 2.0 - } 8 \\
\text { BI }\end{array}$ & & & & & &
\end{tabular}

Notas: $\mathrm{n}=338 ; \chi 2$ MLM: Chi-cuadrado utilizando el método de máxima verosimilitud con errores estándares robustos (MLM); df: grados de libertad; Scaling: factor de ajuste de la corrección de Satorra-Bentler; RMSEA: error cuadrático medio de aproximación; SRMR: residuo estandarizado cuadrático medio, CFI: índice de ajuste comparativo; TLI: Tucker Lewis Index.

Con el fin de evaluar las consistencias internas de todos los modelos analizados, se calcularon las alfas de Cronbach $(\alpha)$ y los coeficientes de omega $(\omega)$. De acuerdo con el contenido de la Tabla 4, se puede afirmar que la versión breve obtuvo valores que están dentro de unos rangos aceptables (Dunn, Baguley \& Brunsden, 2014; Hinton, McMurray \& Brownlow, 2014). Por último, cabe resaltar que no se calcularon las consistencias internas del funcionamiento general para el modelo original, el modelo según el resultado del análisis factorial exploratorio y tampoco para el instrumento breve con 2 factores correlacionados, ya que sus estructuras factoriales no representan un factor global.

Tabla 4.

Consistencias internas de todos los modelos analizados

\begin{tabular}{|c|c|c|c|c|c|c|c|c|}
\hline \multirow[b]{2}{*}{ Modelos competidores } & \multicolumn{4}{|c|}{$\alpha$} & \multicolumn{4}{|c|}{$\omega$} \\
\hline & $\mathrm{AF}$ & FI & $\mathrm{FG}$ & Media de $\alpha$ & $\mathrm{AF}$ & FI & FG & Media de $\omega$ \\
\hline LPFS-BF 2.0 - Original & 0.882 & 0.747 & - & 0.815 & 0.923 & 0.836 & - & 0.880 \\
\hline LPFS-BF 2.0 - EFA & 0.883 & 0.717 & - & 0.800 & 0.906 & 0.751 & - & 0.829 \\
\hline LPFS-BF 2.0 - 8 & 0.844 & 0.739 & - & 0.792 & 0.861 & 0.742 & - & 0.802 \\
\hline LPFS-BF 2.0 - 8 UNI & - & - & 0.835 & 0.835 & - & - & 0.885 & 0.885 \\
\hline LPFS-BF 2.0 - 8 BI & 0.844 & 0.739 & 0.835 & 0.819 & 0.861 & 0.742 & 0.885 & 0.835 \\
\hline
\end{tabular}

Notas: $\mathrm{n}=338$; AF: autofuncionamiento; FI: funcionamiento interpersonal; FG: funcionamiento general; $\alpha$ : alfa de Cronbach; $\omega$ : coeficiente de omega. 


\section{Validez convergente}

En primer lugar, se evaluaron las asociaciones de la edad con las 3 dimensiones del LPFS-BF 2.0 - 8 BI. En el género femenino, la edad tuvo correlaciones bajas con el autofuncionamiento $(\mathrm{rs}=-.283, \mathrm{p}<.001)$ y con el funcionamiento general $(\mathrm{rs}=-.162, \mathrm{p}=$ .027). Por otro lado, el género masculino mostró correlaciones similares: con el autofuncionamiento $(\mathrm{rs}=-.325, \mathrm{p}<.001)$ y con el funcionamiento general $(\mathrm{rs}=-.219, \mathrm{p}=$ $.007)$.

A continuación, se realizó el análisis de validez convergente con las 5 dimensiones del Mini-IPIP. Para ello, se calcularon las correlaciones parciales de Spearman y, con el fin de evaluar posibles diferencias entre géneros, se utilizaron la edad y el nivel educativo como variables de control. Los resultados de este análisis pueden observarse en la Tabla 5. Con respecto a las diferencias más significativas entre géneros, se destaca la responsabilidad con respecto al funcionamiento interpersonal, pues esta relación negativa está menos pronunciada en el género masculino y, por otro lado, la asociación negativa entre extraversión y autofuncionamiento, debido a su menor acentuación en el género femenino. Siguiendo la clasificación de Cohen (1988), se encontró solamente una asociación con un tamaño de efecto grande: en el género femenino, entre autofuncionamiento y neuroticismo. Las demás correlaciones exhibieron tamaños de efecto pequeños y medios.

Tabla 5.

Correlaciones parciales de Spearman entre las dimensiones del modelo final de 8 ítems y el Mini-IPIP, controlando por edad y nivel educativo

\begin{tabular}{lccccccc}
\hline \multirow{2}{*}{ Variables } & \multicolumn{3}{c}{ Femenino } & & \multicolumn{3}{c}{ Masculino } \\
\cline { 2 - 3 } \cline { 6 - 8 } & $\mathrm{AF}$ & $\mathrm{FI}$ & $\mathrm{FG}$ & & $\mathrm{AF}$ & $\mathrm{FI}$ & $\mathrm{FG}$ \\
\hline O - Apertura & $-.242^{* *}$ & $-.337^{* *}$ & $-.323^{* *}$ & & $-.178^{*}$ & $-.334^{* *}$ & $-.281^{* *}$ \\
C - Responsabilidad & $-.246^{* *}$ & $-.292^{* *}$ & $-.326^{* *}$ & & $-.286^{* *}$ & $-.153^{*}$ & $-.258^{* *}$ \\
E - Extraversión & -.099 & $-.129^{*}$ & $-.148^{*}$ & & $-.238^{* *}$ & $-.151^{*}$ & $-.236^{* *}$ \\
A - Amabilidad & $-.180^{* *}$ & $-.475^{* *}$ & $-.365^{* *}$ & & $-.189^{*}$ & $-.388^{* *}$ & $-.319^{* *}$ \\
N - Neuroticismo & $.550^{* *}$ & $.272^{* *}$ & $.494^{* *}$ & & $.454^{* *}$ & $.280^{* *}$ & $.450^{* *}$ \\
\hline
\end{tabular}

Notas: $\mathrm{n}=338 ;$ femenino $=188 ;$ masculino $=150 . * *$ La correlación es significativa en el nivel 0,01 (bilateral). * La correlación es significativa en el nivel 0,05 (bilateral); AF: autofuncionamiento; FI: funcionamiento interpersonal; FG: funcionamiento general.

\section{Invarianza factorial}

A continuación, se realizó el análisis multigrupo por género. En el M2, se igualaron las cargas factoriales entre ambos grupos, en el M3 las cargas factoriales y los interceptos y, en el M4, las cargas factoriales, los interceptos y los residuos. La Tabla 6 muestra los resultados y, como puede observarse, los cambios de los índices se encontraron dentro de unos rangos adecuados con $|\Delta \mathrm{CFI}| \leq 0.010$ según Cheung y Rensvold (2002) e incluso con respecto a los cambios del $\chi 2$ MLM no se han encontrado diferencias significativas. Basado en lo expresado anteriormente, podemos afirmar que el instrumento representa invarianza factorial con relación a los géneros, quiere decir que, independientemente del género del participante, el instrumento evalúa el mismo constructo. 
Tabla 6.

Ajuste del modelo final y comparación de modelos con respecto al género

\begin{tabular}{|c|c|c|c|c|c|c|c|c|c|}
\hline \multirow[t]{2}{*}{ Modelo } & \multicolumn{5}{|c|}{ Ajuste de modelo } & \multicolumn{4}{|c|}{ Comparación de modelos } \\
\hline & S-B $\chi 2^{\text {MLM }}$ & $d f$ & $\chi^{2 / d f}$ & Scaling & CFI & $\Delta \mathrm{S}-\mathrm{B} \chi 2^{\mathrm{MLM}}$ & $\Delta \mathrm{df}$ & $p$ & $\triangle \mathrm{CFI}$ \\
\hline $\begin{array}{l}\text { M1: } \\
\text { Configural }\end{array}$ & 19.686 & 24 & 0.820 & 1.216 & 1.000 & & & & \\
\hline M2: Débil & 35.106 & 37 & 0.949 & 1.184 & 1.000 & 15.420 & 13 & 0.282 & -0.000 \\
\hline M3: Fuerte & 38.339 & 42 & 0.913 & 1.179 & 1.000 & 03.233 & 5 & 0.664 & -0.000 \\
\hline M4: Estricto & 52.727 & 50 & 1.055 & 1.169 & 0.997 & 14.388 & 8 & 0.072 & -0.003 \\
\hline
\end{tabular}

Notas: $\mathrm{n}=338$; femenino $=188 ;$ masculino $=150 ; \chi 2$ MLM - Chi-cuadrado utilizando el método de máxima verosimilitud con errores estándares robustos (MLM); df: Grados de libertad; Scaling: factor de ajuste de la corrección de Satorra-Bentler; RMSEA: error cuadrático medio de aproximación; CFI: índice de ajuste comparativo.

\section{Estadística descriptiva}

La Tabla 7 muestra la estadística descriptiva del modelo final según géneros. Teniendo en cuenta el índice de límites aceptables de asimetría y curtosis de \pm 2 (Hinton et al., 2014), se puede afirmar que todos los ítems están leve a moderadamente sesgados positivamente, pero no hay valores atípicos extremos en la muestra (asimetría máx. $=1.202$, curtosis máx. $=-1.019$ ).

Tabla 7.

Estadística descriptiva del modelo final

\begin{tabular}{|c|c|c|c|c|c|c|c|c|c|c|c|}
\hline \multirow{2}{*}{ Dimensión } & \multirow{2}{*}{ Ítem } & \multicolumn{5}{|c|}{ Femenino } & \multicolumn{5}{|c|}{ Masculino } \\
\hline & & $M$ & $D E$ & Mdn & Asimetría & Curtosis & $M$ & $D E$ & Mdn & Asimetría & Curtosis \\
\hline \multicolumn{12}{|c|}{ Auto funcionamiento } \\
\hline & LPFS_01 & 1.021 & 1.114 & 1.000 & 0.836 & -0.272 & 1.127 & 1.255 & 1.000 & 0.896 & -0.268 \\
\hline & LPFS_03 & 1.596 & 1.235 & 1.000 & 0.306 & -0.965 & 1.360 & 1.177 & 1.000 & 0.482 & -0.748 \\
\hline & LPFS_04 & 0.947 & 1.117 & 1.000 & 1.202 & -0.798 & 1.153 & 1.252 & 1.000 & 0.874 & -0.300 \\
\hline & LPFS_05 & 1.282 & 1.184 & 1.000 & 0.677 & -0.441 & 1.267 & 1.251 & 1.000 & 0.634 & -0.736 \\
\hline \multicolumn{12}{|c|}{ Funcionamiento interpersonal } \\
\hline & LPFS_07 & 0.915 & 0.955 & 1.000 & 0.828 & -0.120 & 1.307 & 1.111 & 1.000 & 0.489 & -0.717 \\
\hline & LPFS_08 & 1.383 & 1.153 & 1.000 & 0.472 & -0.646 & 1.440 & 1.156 & 1.000 & 0.314 & -0.863 \\
\hline & LPFS_09 & 1.330 & 1.146 & 1.000 & 0.439 & -0.669 & 1.567 & 1.223 & 1.000 & 0.273 & -1.019 \\
\hline & LPFS_12 & 0.947 & 1.001 & 1.000 & 0.646 & -0.678 & 1.220 & 1.086 & 1.000 & 0.621 & -0.573 \\
\hline
\end{tabular}

Notas: $\mathrm{n}=338 ;$ femenino $=188 ;$ masculino $=150 ;$ M: Media; DE: desviación estándar; Mdn: Mediana. 


\section{Discusión}

Con el objetivo de realizar la adaptación de la Level of Personal Functioning Scale Brief Form 2.0, se realizó un análisis factorial exploratorio, una reducción de ítems, un análisis factorial confirmatorio de varias estructuras factoriales, una comprobación de la validez convergente y un análisis multigrupo para determinar la invarianza factorial por género.

De acuerdo con los valores de corte que $\mathrm{Hu}$ y Bentler (1999) sugieren para la evaluación de modelos de ecuaciones estructurales, se observó que estos no se encontraron en unos rangos adecuados, por lo que varios ítems tuvieron que ser eliminados. Tras la medida de purificación, todos los índices de ajuste mostraban valores satisfactorios, lo cual permite constatar la validez del instrumento adaptado. Asimismo, los resultados del análisis de multigrupo constatan que el instrumento evalúa, independientemente del género de la persona, el mismo constructo. Este resultado facilita la posibilidad de utilizar esta técnica en futuras investigaciones que tengan como objetivo determinar diferencias de grupos (Byrne, 2016). Por otro lado, es importante resaltar que todos los índices de ajuste, los cuales fueron obtenidos en el presente estudio, representan una discrepancia mínima entre el modelo hipotetizado y los datos observados, ya que no se han utilizado covarianzas residuales entre los ítems.

Aun así, debe tenerse en cuenta que se utilizó la misma muestra para el análisis factorial exploratorio y para el análisis factorial confirmatorio. Aunque este procedimiento pueda conducir hacia la reducción del poder de confirmación (Fokkema \& Greiff, 2017), es importante mencionar que el análisis factorial exploratorio del presente estudio exhibió los mismos resultados que obtuvieron Bach y Hutsebaut (2018), quiere decir que no encontró una estructura factorial nueva, sino que reconfirmó una estructura ya establecida.

El segundo objetivo del presente estudio consistió en el análisis de la estructura factorial del instrumento y, de acuerdo con los índices de ajuste encontrados, se determinó que tiene una estructura bidimensional. Es importante resaltar este hallazgo, ya que permite no solo la utilización de sus dos factores, autofuncionamiento y funcionamiento interpersonal, sino también el factor de funcionamiento general (Reise, Moore \& Haviland, 2010). Asimismo, se resalta que el análisis de validez convergente mostró asociaciones significativas entre estas dimensiones y todos los rasgos de la personalidad, lo cual está en consonancia con otros estudios (Hopwood, Good \& Morey, 2018; McCabe \& Widiger, 2020).

Aunque la versión adaptada de la Level of Personal Functioning Scale - Brief Form 2.0 muestra valores psicométricos adecuados, debe enfatizarse que estos fueron obtenidos a través de una reducción, por lo que comprende solamente 8 ítems y no 12 como la versión en lengua inglesa. Ya en el estudio original de la primera versión del instrumento se advirtieron dificultades con determinados ítems (Hutsebaut, Feenstra \& Kamphuis, 2016), por lo que un estudio posterior de Weekers et al. (2019) recurrió a la utilización de una covarianza residual y una carga factorial cruzada (cross-loading), quiere decir que los autores asignaron un ítem, simultáneamente, a 2 factores. A pesar de estas medidas, los autores no pudieron alcanzar los valores propuestos por Hu y Bentler (1999). 
Por otro lado, se enfatiza en que la presente versión adaptada recurrió a la utilización de una escala Likert de 5 puntos, aunque los autores del instrumento original habían utilizado una de 4 valores. Esa decisión se basó en que, durante la prueba piloto del estudio, un elevado número de participantes había manifestado su deseo de marcar una respuesta de valor intermedio. En este punto, debe tenerse en cuenta que Garland (1991) mostró que el sesgo de deseabilidad social puede reducirse eliminando el punto medio de una escala Likert y que retenerlo podría distorsionar los resultados. Por otro lado, eliminar el punto neutro también introduce una elección forzada en la escala (Allen \& Seaman, 2007), ya que una persona encuestada podría verse obligada a declarar una posición en lugar de permanecer neutral, lo que en algunos casos políticos y delicados puede no ser deseable (Leung, 2011). Con relación a las propiedades psicométricas, Leung (2011) no encontró diferencias con respecto a la utilización o no utilización de un punto neutro. De esta manera, se observó en el presente estudio que todos los valores psicométricos se encontraron en unos rangos aceptables. Además, la estadística descriptiva evidenció que la decisión de utilizar una escala Likert de 5 puntos no aparenta haber causado un posible sesgo hacia las opciones intermedias, ya que las medias y medianas se encontraron notablemente por debajo del punto neutro.

Además de lo mencionado, deben observarse las siguientes limitaciones: el tamaño muestral del presente estudio puede considerarse relativamente pequeño. Debido al muestreo no probabilístico, debe tenerse en cuenta también que los valores de la estadística descriptiva no son representativos, por lo que no deberían utilizarse sus valores con el fin de extraer determinadas conclusiones. Además, la muestra se tomó durante las restricciones de la pandemia de la COVID-19, lo cual pudo haber influido en la obtención de los valores. Finalmente, se destaca que se utilizaron solamente los rasgos de la personalidad para el análisis de validez convergente y que, para poder reportar los índices correspondientes a un Test-Retest, se requiere un estudio longitudinal, el cual no se realizó en el presente estudio.

Estas circunstancias conducen a la necesidad de futuros estudios para poder consolidar la confiabilidad y la estructura bifactorial del instrumento, y para poder predecir otros constructos como, por ejemplo, determinada sintomatología o trastornos de la personalidad que pudieran ser de interés.

\section{Referencias}

Allen, I. E., \& Seaman, C. A. (2007). Likert scales and data analyses. Quality Progress, 40(7), 64-65.

American Psychiatric Association. (2000). Diagnostic criteria from dsM-iV-tr. American Psychiatric Pub.

American Psychiatric Association. (2013). Diagnostic and statistical manual of mental disorders (DSM-5®). American Psychiatric Pub.

Bach, B., \& Hutsebaut, J. (2018). Level of Personality Functioning Scale-Brief Form 2.0: Utility in Capturing Personality Problems in Psychiatric Outpatients and Incarcerated Addicts. Journal of Personality Assessment, 100(6), 660-670. doi: https://doi.org/10.1080/00223891.2018.1428984 
Bender, D. S., Morey, L. C., \& Skodol, A. E. (2011). Toward a Model for Assessing Level of Personality Functioning in DSM-5, Part I: A Review of Theory and Methods. Journal of Personality Assessment, 93(4), 332-346. doi: https://doi.org/10.1080/00223891.2011.583808

Bernstein, R. F. (1998). Reconceptualizing Personality Disorder Diagnosis in the DSM-V : The Discriminant Validity Challenge. Clinical Psychology: Science and Practice, 5(3), 333-343. doi: https://doi.org/10.1111/j.1468-2850.1998.tb00153.x

Byrne, B. M. (2016). Structural Equation Modeling with AMOS. New York: Routledge. doi: https://doi.org/10.4324/9781315757421

Calabrese, W. R., \& Simms, L. J. (2014). Prediction of daily ratings of psychosocial functioning: Can ratings of personality disorder traits and functioning be distinguished? Personality Disorders: Theory, Research, and Treatment, 5(3), 314322. doi: https://doi.org/10.1037/per0000071

Cheung, G. W., \& Rensvold, R. B. (2002). Evaluating Goodness-of-Fit Indexes for Testing Measurement Invariance. Structural Equation Modeling: A Multidisciplinary Journal, 9(2), 233-255. doi: https://doi.org/10.1207/S15328007SEM0902_5

Cohen, J. (1988). Statistical power analysis for the behavioral sciences ( $2^{\circ} \mathrm{Ed}$.). New Jersey: Lawrence Erlbaum Associates.

Core Team. (2020). R: A language and environment for statistical computing. Vienna, Austria. Recuperado de https://www.r-project.org/

Costello, A., \& Osborne, J. (2005). Best practices in exploratory factor analysis: Four recommendations for getting the most from your analysis. Practical Assessment, Research, and Evaluation, 10(7), 1-9. doi: https://doi.org/10.7275/jyj1-4868

de la Parra, G., Undurraga, C., Crempien, C., Valdés, C., Dagnino, P., \& Gómez-Barris, E. (2018). Estructura de Personalidad en Pacientes con Depresión: Adaptación de un Instrumento y Resultados Preliminares. Psykhe, 27(2), 1-20. doi: https://doi.org/10.7764/psykhe.27.2.1133

Dunn, T. J., Baguley, T., \& Brunsden, V. (2014). From alpha to omega: A practical solution to the pervasive problem of internal consistency estimation. British Journal of Psychology, 105(3), 399-412. doi: https://doi.org/10.1111/bjop.12046

Ehrenthal, J., Dinger, U., Horsch, L., Komo-Lang, M., Klinkerfuß, M., Grande, T., \& Schauenburg, H. (2012). Der OPD-Strukturfragebogen (OPD-SF): Erste Ergebnisse $\mathrm{zu}$ Reliabilität und Validität. Psychotherapie, Psychosomatik, Medizinische Psychologie, 62(01), 25-32. doi: https://doi.org/10.1055/s-0031-1295481

Ehrenthal, J., Dinger, U., Schauenburg, H., Horsch, L., Dahlbender, R. W., \& Gierk, B. (2015). Entwicklung einer Zwölf-Item-Version des OPD-Strukturfragebogens (OPDSFK)/ Development of a 12-item version of the OPD-Structure Questionnaire (OPDSQS). Zeitschrift Für Psychosomatische Medizin Und Psychotherapie, 61(3), 262274. doi: https://doi.org/10.13109/zptm.2015.61.3.262

Fabrigar, L. R., Wegener, D. T., MacCallum, R. C., \& Strahan, E. J. (1999). Evaluating the use of exploratory factor analysis in psychological research. Psychological Methods, 4(3), 272-299. doi: https://doi.org/10.1037/1082-989X.4.3.272

Fokkema, M., \& Greiff, S. (2017). How Performing PCA and CFA on the Same Data Equals Trouble. European Journal of Psychological Assessment, 33(6), 399-402. doi: https://doi.org/10.1027/1015-5759/a000460 
Garland, R. (1991). The mid-point on a rating scale: Is it desirable. Marketing Bulletin, 2(1), 66-70.

Hair, J. F., Black, W. C., Babin, B. J., \& Anderson, R. E. (2010). Multivariate data analysis ( $7^{\circ}$ ed.). New York: Prentice Hall.

Hentschel, A. G., \& Livesley, W. J. (2013). The General Assessment of Personality Disorder (GAPD): Factor Structure, Incremental Validity of Self-Pathology, and Relations to DSM-IV Personality Disorders. Journal of Personality Assessment, 95(5), 479-485. doi: https://doi.org/10.1080/00223891.2013.778273

Hinton, P., McMurray, I., \& Brownlow, C. (2014). SPSS Explained (2 ${ }^{\circ}$ ed.). New York: Routledge. doi: https://doi.org/10.4324/9781315797298

Hofmann, R. J. (1978). Complexity And Simplicity As Objective Indices Descriptive Of Factor Solutions. Multivariate Behavioral Research, 13(2), 247-250. doi: https://doi.org/10.1207/s15327906mbr1302_9

Hopwood, C. J., Good, E. W., \& Morey, L. C. (2018). Validity of the DSM-5 Levels of Personality Functioning Scale-Self Report. Journal of Personality Assessment, 100(6), 650-659. doi: https://doi.org/10.1080/00223891.2017.1420660

Hopwood, C. J., Malone, J. C., Ansell, E. B., Sanislow, C. A., Grilo, C. M., McGlashan, T. H., ... Morey, L. C. (2011). Personality Assessment in DSM-5: Empirical Support for Rating Severity, Style, and Traits. Journal of Personality Disorders, 25(3), 305320. doi: https://doi.org/10.1521/pedi.2011.25.3.305

Hopwood, C. J., Schade, N., Krueger, R. F., Wright, A. G. C., \& Markon, K. E. (2013). Connecting DSM-5 Personality Traits and Pathological Beliefs: Toward a Unifying Model. Journal of Psychopathology and Behavioral Assessment, 35(2), 162-172. doi: https://doi.org/10.1007/s10862-012-9332-3

Hopwood, C. J., Thomas, K. M., Markon, K. E., Wright, A. G. C., \& Krueger, R. F. (2012). "DSM-5 personality traits and DSM-IV personality disorders": Correction to Hopwood et al. (2012). Journal of Abnormal Psychology, 121(2), 432-432. doi: https://doi.org/10.1037/a0028553

Hu, L., \& Bentler, P. M. (1999). Cutoff criteria for fit indexes in covariance structure analysis: Conventional criteria versus new alternatives. Structural Equation Modeling: A Multidisciplinary Journal, 6(1), 1-55. doi: https://doi.org/10.1080/10705519909540118

Hutsebaut, J., Feenstra, D. J., \& Kamphuis, J. H. (2016). Development and Preliminary Psychometric Evaluation of a Brief Self-Report Questionnaire for the Assessment of the DSM-5 level of Personality Functioning Scale: The LPFS Brief Form (LPFSBF). Personality Disorders: Theory, Research, and Treatment, 7(2), 192-197. doi: https://doi.org/10.1037/per0000159

Kernberg, O. F. (2012). Overview and critique of the classification of personality disorders proposed for DSM-V. Schweizer Archiv Für Neurologie Und Psychiatrie, 163(7), 234-238. doi: https://doi.org/10.4414/sanp.2012.00110

Korkmaz, S., Goksuluk, D., \& Zararsiz, G. (2014). MVN: An R Package for Assessing Multivariate Normality. The $R$ Journal, 6(2), 151-162. doi: https://doi.org/10.32614/RJ-2014-031

Laireiter, A. (2013). Diagnostik in der Psychotherapie. Springer-Verlag. 
Leising, D., \& Zimmermann, J. (2011). An Integrative Conceptual Framework for Assessing Personality and Personality Pathology. Review of General Psychology, 15(4), 317330. doi: https://doi.org/10.1037/a0025070

Lenzenweger, M. F., Clarkin, J. F., Kernberg, O. F., \& Foelsch, P. A. (2001). The Inventory of Personality Organization: Psychometric properties, factorial composition, and criterion relations with affect, aggressive dyscontrol, psychosis proneness, and selfdomains in a nonclinical sample. Psychological Assessment, 13(4), 577-591. doi: https://doi.org/10.1037/1040-3590.13.4.577

Leung, S.-O. (2011). A Comparison of Psychometric Properties and Normality in 4-, 5-, 6-, and 11-Point Likert Scales. Journal of Social Service Research, 37(4), 412-421. doi: https://doi.org/10.1080/01488376.2011.580697

Levy, K., \& Clarkin, J. F. (2003). The influence of client variables on psychotherapy. En M. Lambert (Ed.), Handbook of Psychotherapy and Behavior Change (Vol. 5, pp. 194226). New York: Wiley \& Sons.

Leys, C., Klein, O., Dominicy, Y., \& Ley, C. (2018). Detecting multivariate outliers: Use a robust variant of the Mahalanobis distance. Journal of Experimental Social Psychology, 74, 150-156. doi: https://doi.org/10.1016/j.jesp.2017.09.011

Livesley, W. J. (1998). Suggestions for a Framework for an Empirically Based Classification of Personality Disorder. The Canadian Journal of Psychiatry, 43(2), 137-147. doi: https://doi.org/10.1177/070674379804300202

Luyten, P., \& Blatt, S. J. (2011). Integrating theory-driven and empirically-derived models of personality development and psychopathology: A proposal for DSM V. Clinical Psychology Review, 31(1), 52-68. doi: https://doi.org/10.1016/j.cpr.2010.09.003

Mardia, K. V. (1970). Measures of multivariate skewness and kurtosis with applications. Biometrika, 57(3), 519-530. doi: https://doi.org/10.1093/biomet/57.3.519

McCabe, G. A., \& Widiger, T. A. (2020). A comprehensive comparison of the ICD-11 and DSM-5 section III personality disorder models. Psychological Assessment, 32(1), 7284. doi: https://doi.org/10.1037/pas0000772

Morey, L. C., Berghuis, H., Bender, D. S., Verheul, R., Krueger, R. F., \& Skodol, A. E. (2011). Toward a Model for Assessing Level of Personality Functioning in DSM-5, Part II: Empirical Articulation of a Core Dimension of Personality Pathology. Journal of Personality Assessment, 93(4), 347-353. doi: https://doi.org/10.1080/00223891.2011.577853

Reise, S. P., Moore, T. M., \& Haviland, M. G. (2010). Bifactor Models and Rotations: Exploring the Extent to Which Multidimensional Data Yield Univocal Scale Scores. Journal of Personality Assessment, 92(6), 544-559. doi: https://doi.org/10.1080/00223891.2010.496477

Revelle, W. (2019). Psych: Procedures for personality and psychological research. Recuperado de https://cran.r-project.org/package=psych Version $=1.9 .12$

Rosseel, Y. (2012). lavaan: An R Package for Structural Equation Modeling. Journal of Statistical Software, 48(2), 1-36. https://doi.org/10.18637/jss.v048.i02

Satorra, A., \& Bentler, P. M. (2000). A Scaled Difference Chi-Square Test Statistic for Moment Structure Analysis. SSRN Electronic Journal, 66(4), 507-514. doi: https://doi.org/10.2139/ssrn.199064 
Simkin, H., Borchardt Dutera, L., \& Azzollini, S. (2020). Evidencias de validez del Compendio Internacional de Ítems de Personalidad Abreviado. Liberabit: Revista Peruana de Psicología, 26(1), 1-23. https://doi.org/10.24265/liberabit.2020.v26n1.02

Sleep, C. E., Wygant, D. B., \& Miller, J. D. (2018). Examining the Incremental Utility of DSM-5 Section III Traits and Impairment in Relation to Traditional Personality Disorder Scores in a Female Correctional Sample. Journal of Personality Disorders, 32(6), 738-752. doi: https://doi.org/10.1521/pedi_2017_31_320

Stieglitz, R.-D. (2014). Psychometrische Verfahren in der Psychotherapie. Verhaltenstherapie, 24(1), 56-65.

Stover, J. B., \& Bruno, F. E. (2019). Estudios psicométricos del Level Of Personality Function Scale en adultos de Buenos Aires. XI Congreso Internacional de Investigación y Práctica Profesional En Psicología. XXVI Jornadas de Investigación. XV Encuentro de Investigadores En Psicología del Mercosur. I Encuentro de Investigación de Terapia Ocupacional. I Encuentro de Musicoterapia. Facultad de Psicología-Universidad de Buenos Aires.

Venables, W. N., \& Ripley, B. D. (2002). Modern Applied Statistics with S. New York: Springer. doi: https://doi.org/10.1007/978-0-387-21706-2

Verheul, R., Andrea, H., Berghout, C. C., Dolan, C., Busschbach, J. J. V., van der Kroft, P. J. A., ... Fonagy, P. (2008). Severity Indices of Personality Problems (SIPP-118): Development, factor structure, reliability, and validity. Psychological Assessment, 20(1), 23-34. doi: https://doi.org/10.1037/1040-3590.20.1.23

Weekers, L. C., Hutsebaut, J., \& Kamphuis, J. H. (2019). The Level of Personality Functioning Scale-Brief Form 2.0: Update of a brief instrument for assessing level of personality functioning. Personality and Mental Health, 13(1), 3-14. doi: https://doi.org/10.1002/pmh.1434

World Health Organization. (1993). The ICD-10 classification of mental and behavioural disorders: diagnostic criteria for research (Vol. 2). World Health Organization.

Yu, C.-Y. (2002). Evaluating cutoff criteria of model fit indices for latent variable models with binary and continuous outcomes. Los Ángeles: University of California.

Zimmermann, J., Benecke, C., Bender, D. S., Skodol, A. E., Krueger, R. F., \& Leising, D. (2013). Persönlichkeitsdiagnostik im DSM-5. Psychotherapeut, 58(5), 455-465. doi: https://doi.org/10.1007/s00278-013-1009-1

Contribución de los autores: a) Concepción y diseño del trabajo; b) Adquisición de datos; c) Análisis e interpretación de datos; d) Redacción del manuscrito; e) revisión crítica del manuscrito.

C. S. ha contribuido en a, b, c, d, e.

Editora científica responsable: Dra. Cecilia Cracco. 